\title{
Contribuição para o conhecimento da fauna de pro- tozoarios do Brazil
}

\author{
IV \\ pelo \\ Dr. ARISTIDES MARQUES DA CUNHA
}

(Conı a estampa 3).

\begin{abstract}
O atual trabalho representa nova contribuição para o conhecimento da fauna de protozoarios do Brazil, assunto que ha tempos prende a nossa atenção e que já tem sido objeto de notas anteriores.
\end{abstract}

Neste trabalho nos ocuparemos com os protozoarios de agua doce e salôbra; os protozoarios marinhos são presentemente objeto de estudo em colaboração com o Dr. GOMES DE FARIA e o resultado dessas pesquizas será opurtunamente publicado em memoria especial.

Em nossa primeira contribuição, fizemos a resenha dos trabalhos existentes sobre o assunto. Após a publicação de nosso trababalho, apareceu um artigo de WAILES sobre os rizopodes da America do Norte e do Sul no qual vêm mencionadas 51 especies encontradas no Rio de Janeiro, 38 das quaes ainda não haviam sido assinaladas no Brazil. $O$ autor pesquizou os rizopodes testaceos encontrados em musgo e esfagno de fórma que seu trabalho vem completar um ponto que havia sido descurado no nosso.
Dos arredores de. Manguinhos temos examinado grande numero de amostras de agua doce e salôbra e, ás especies mencionadas em nosso primeiro trabalho pudemos acrecentar mais 22 que constam da lista que adiante transcrevemos:

1. Mastigamoeba aspera SCHULTZE 1875.

2. Hexamitus crassus KLEBS, 1892.

3. Petalomonas angusta KLEBS, 1892.

4. Petalomonas sexlobata KLEBS, 1892.

5. Dinema griseolum PERTY, 1852.

6. Tropidomonas rotans CUNHA, 1915.

7. Coccomonas orbicularis STEIN, 1878.

8. Volvulina steinii PLAYFAIR, 1915.

9. Hemidinium nasutum STEIN, 1883.

10. Chilodon dubius MAUPAS, 1883.

11. Leptopharynx costatus MERMOD, 1914.

12. Epalxis mirabilis ROUX, 1899.

13. Saprodinium dentatum LAUTERBORN, 1908.

14. Plagiopyla nasuta STEIN, 1860.

15. Metopus nasutus CUNHA, 1915.

16. Metopus caudatus CUNHA, 1915. 
17. Tropidoactratus accuminatus LEV, 1894.

18. Spirorhynchus verrucosus CUNHA, 1915.

19. Strombidium stylifer LEVANDER, 1894.

20. Stichochaeta pediculiforme $\mathrm{COHN}$, 1866.

21. Diophrys appendiculatus COHN, 1866.

22. Uronychia transfuga O. F. MUELLER, 1777.

Em excursão que em companhia do Dr. L. TRAVASSOS, fizemos nos arredores de Angra dos Reis, tivemos oportunidade de observar numerosas amostras de agua doce e pudemos verificar a presença de 64 especies de protozoarios, algumas das quaes ainda não haviamos observado, sendo uma, Euglypha cristata, LEIDY 1874, ainda não assinalada na nossa fauna.

Damos em seguida a lista das especies encontradas :

1. Amoeba proteus PALL. 1766.

2. Arcella valgaris EHRB, 1830.

3. Arcella brasiliensis CUNHA, 1913.

4. Difflugia accuminata EHRB, 1830.

5. Difflugia oblonga EHRB. 1831.

Esta especie já havia sido assinalada em trabalho anterior sobo nome de Difflugia piriformis PERTY 1849. Esse nome deve porém ser substituido por Difflugia oblonga EHRB, em virtude da lei de prioridade, ficando aquele como sinonimo.

6. Difflugia constricta EHRB, 1841.

7. Difflugia urceolata CARTER, $181,4$.

8. Difflugia curvicaulis PENARD, 1899.

9. Centropyxis aculeata (EHRB, 1830).

10. Centropyxis impressa (DADAY, 1905).

11. Lecquereusia spiralis (EHRB, 1830).

12. Nebela collaris (EHRB. 1848).

13. Quadrula symetrica (WALLICH, 1863).

14. Euglypha alveolata DUJ. 1841.

15. Euglypha brachiata LEIDY, 1878.

16. Euglypha cristata LEIDY, 1874.

17. Trinema euchelys (EHRB, 1838).

18. Trinema lineare (PENARD, 1890).

19. Cyphoderia ampulla (EHRB, 1840).

20. Clathrulina elegans (CIENK, 1867).
21. Dendromonas laxa (KENT, 1881).

22. Euglena viridis SCHRANK. 1780.

23. Euglena deses EHRB, 1833.

24. Euglena tripteris (DUJ, 1841).

25. Crumenula ovum (EHRB, 1840).

26. Crumenula globosa (FRANCE, 1893).

27. Phacus pleuronectes (O. F. MUELLER, 1773).

28. Phacus parvula KLEBS, 1883.

29. Trachelomonas volvocina $\mathrm{EHRB}, 1831$.

30. Trachelomonas cilindrica, EHRB 1833.

31. Trachelomonas obtusa, PALMER, 1905.

32. Astasia curvata (KLEBS, 1883).

33. Peramena trichophorum (EHRB, 1830).

34. Entosyphon sulcatum (DUJ, 1841).

35. Anisonema acinus (DUJ, 1841).

36 Heteronema acus (EHRB, 1840).

37. Cryptomonas ovata EHRB, 1831.

38. Chilomonas paramecium EHRB, 1831.

39. Chilomonas prowazeki CUNHA, 1913.

40. Gymnodinium fuscum (EHRB, 1833).

41. Glenodinium cinctum EHRB, 1835.

42. Lacrymaria olor (O. F. MUELLER, 1786.

43. Prorodon teres EHRB, 1833.

44. Coleps hirtus (O. F. MUELLER, 1786).

45. Mesodinium acarus STEIN, 1862.

46. Lionotus fasciola (O. F. MUELLER, 1786).

47. Loxodes rostrum (O. F. MUELLER, 1786).

48. Dileptus anser (O. F. MUELLER, 1786).

49. Loxocephalus granulosus KENT, 1881.

50. Colpoda cucullus O. F. MUELLER, 1786.

51. Frontonia leucas (EHRB, 1833.

52. Frontonia accuminata (EHRB, 1831).

53. Cinetochilum margaritaceum (EHRB, 1831).

54. Drepanomonas dentata (FRES, 1858).

55. Ureocentrum turbo (O. F. MUELLER, 1786).

56. Lembodion bullinum (O. F. MUELLER, 1786).

57. Cyclidiuır. glaucoma (O. F. MUELLER, 1786). 
58. Spirostomum ambiguum (EHRB, 1830).

59. Spirostomum teres CL. \& LACH. 1858.

60. Halteria grandinella (O. F. MUELLER, 1786).

61. Strombilidium gyrans (STOKES, 1887).

62. Oxytricha platystoma EHRB, 1831.

63. Euplotes patella (O. F. MUELLER, 1773).

64. Vorticella citrina (O. F. MUELLER, 1773).

Em material colecionado pelo Dr. A. NEIVA durante uma viajem pelo Brazil central, encontrámos as especies que constam da lista abaixo:

Peixe, Bahia (Municipio de Remanso);

1. Centropyxis aculeata (EHRB, 1830)

2. Trinema enchelys (EHRB, 1833).

3. Phacus pleuronectes (O. F. MUELLER, 1773).

Lagôa de Parnaguá, Piauhy (Municipio de Parnaguá);

1. Difflugia limnetica LEVANDER 1900.

2. Centropyxis aculeata (EHRB, 1830).

3. Euglypha alveolata DUJ, 1841.

4. Englena fusca KLEBS, 1883.

5. Phacus longicauda (EHRB, 1830).

6. Entosyphon sulcatum (DUJ, 1841).

7. Chilomonas paramacium EHRB, 1831.

8. Coleps hirtus (O. F. MUELLER 1786).

\section{Tropidomonas CUNHA 1915.}

Flajelado de corpo rijido, provido de saliencias lonjitudinaes em forma de cristas enroladas em espiral. Com um flajelo.

Esse genero, cuja diagnose já foi dada em nota previa, deve ser incluido na familia Peranematidae. É representado até agora pela especie unica que passamos a descrever.

\section{Tropidomonas rotans CUNHA 1915.}

Corpo rijido, elipsoide, possuindo em sua superficie 10 saliencias lonjitudinaes em forma de cristas, levemnente enroladas em espiral. O corpo é incolôr, hialino e apresenta grande quantidade de corpusculos de paramilo, sobretudo na parte anterior. $O$ flajelo que é unico, acha-se situado na extremidade anterior do corpo e tem mais ou menos o comprimento deste.

O flajelado move-se, descrevendo uma linha sinuosa e é então animado de movimento de rotação em torno do eixo lonjitudinal. Em preparados córados, observa-se que o nucleo, situado na parte posterior do corpo, apresenta um cariosoma redondo central, cercado de abunciante cromatina periferica, disposta em granulações.

Dimensões : comprimente $30 \mu$, largura $20 \mu$.

Habitat: Encontrada em agua doce, proveniente dos arredores de Manguinhos.

\section{Metopus CL. e LACH. 1858.}

O genero Metopus, criado por CLAPAREDE e LACHMANN com uma unica especie, Metopus sigmoidis, foi mais tarde enriquecido com especies novas principalmente por LEVANDER. Nem todos os autores, porém admitem as especies posteriormente incluidas nesse genero e consideram taes especies como formas de Metopus sigmoidis nas quaes, a maior ou menor torção do corpo fez com que alguns autores as tomassem como especies independentes.

Essa opinião não nos parece livre de objeção, pois algumas das especies em questão, além do maior ou menor gráo de torção do corpo, apresentam outros caracteres diferenciaes, como se dá com o Metopus piriformis que apresenta a extremidade posterior acuminada, ao passo que ela é arredondada no Metopus sigmoidis.

No decorrer de nossas pesquizas, tivemos ocasião de observar duas especies desse genero, contra as quaes não se aplica a objeção levantada por esses autores, pois essas especies não se diferenciam de Metopus sigmoidis pela torção do corpo e sim por caracteres morfolojicos considerados por todos como especificos e largamente aplicados na distinção das especies de outros generos, Essas especies, de que já demos a diagnose em nota previa, são encontradas, uma em agua doce e outra em agua salôbra. 


\section{Metopus nasutus CUNHA, 1915.}

Corpo alongado, e mais ou menos achatado no sentido dorso-ventral. Parte anterior torcido sobre a face ventral da direita para a esquerda. Parte posterior arredundada. $O$ peristoma, levemente obliquo de cima para baixo e da esquerda para a direita, prolongase da extremidade anterior ao meio do corpo. Boca situada na extremidade posterior do peristoma. O corpo é revestido de cilios finos dispostos em linhas lonjitudinaes. $\mathrm{Na}$ borda esquerda do peristoma, constituindo a zona adoral, existe uma fileira de cilios mais longos e mais espessos que os demais. Na extremidade posterior do corpo, existem cilios longos e finos.

Da extremidade anterior do corpo, parte um prolongamento cilindrico, flexivel, desprovido de de cilios, com cerca de $1 / 3$ do comprimento do corpo. Este prolongamento dirijido geralmente para tráz mantem-se pendente ao longo do corpo. Macronucleo elipsoide, junto ao qual ha um micronucleo. Vacuolo contratil unico situado na extremidade posterior do corpo.

Dimensões : comprimento $100 \mu$, largura $30 \mu$, comprimento do prolongamento anterior 30-40 $\mu$.

Pela forma e dimensões do corpo esta especie se aproxima de Melopus sigmoidis da qual facilmente se diferencia pelo prolongamento anterior.

Habitot: Encontrado em agua doce nos arredores de Manguinhos.

\section{Metopus caudatus CUNHA 1915.}

Corpo alongado e achatado no sentido dorso-ventral. Parte anterior torcida sobre a face ventral da direita para a esquerda. Parte posterior gradualmente estreitada e termi nada em um prolongamento caudal. Operistoma obliquo de cima para baixo e da esquerda para a direita, prolonga-se da extremidade anterior ao meio do corpo. $\mathrm{O}$ peristoma é mais obliquo que na especie antericr e é lijeiramente curvo com a concavidade voltada para baixo e para a esquerda.

O corpo é revestido de cilios finos, dispostos em linhas lonjitudinaes, sendo que no prolongamento caudal os cilios se tornam mais espaçados, á medida que se aproximam da extremidade. $\mathrm{Na}$ borda esquerda do peristoma, constituindo a zona adoral, existe uma fileira de cilios mais longos e mais espessos que os demais.

Macronucleo elipsoide junto ao qual ha um micronucleo.

Vacuolo contratil, unico, situado na parte posterior, na base de prolongamento caudal.

Dimensões: comprimento 90-100 $\mu$, comprimento do prolongamento caudal $30 \mu$, largura $30 \mu$.

Esta especie tambem se aproxima do Metopus sigmoidis, da qual se diferencia pelo prolongamento caudal.

Habitat: Encontrado em agua salôbra, nos arredores de Manguinhos.

\section{Spirorhynchus CUNHA 1915.}

Heterotricha provido de um prolongamento anterior em forma de tromba. Peristoma constituido por um sulco enrolado em espiral em torno desse prolongamento. Zona adoral formada por uma fileira de cilios mais espessos que os que revestem o corpo, tambem enrolados em espiral, em torno da tromba, acompanhando a borda esquerda do peristoma. Boca situada na base da tromba na extremidade posterior do peristoma. Corpo, com exceção da zona adoral, uniformemente ciliado.

Este genero, do qual já demos'descrição em nota previa, deve ser incluido na familia Plagiotomidae, ; distingue-se dos demais pela situação do peristoma em um prolongamento anterior. É representado até agora pela especie unica que passamos a descrever.

\section{Spirorhynchus verrucosus CUNHA 1915.}

Corpo fusiforme, terminado anteriormente em um prolongamento em forma de tromba e posteriormente em um prolongamento caudal longo e ponteagudo. Peristoma em forma de sulco, enrolado em espiral em torno da tromba. Zona adoral constituida por uma fileira de cilios mais espessos que 
os que revestem o corpo, tambem enrolado em espiral em redor da tromba, acompanhando a borda esquerda do peristoma. Boca situada na extremidade posterior do peristoma. $O$ corpo é uniformemente revestido de cilios finos e longos, dispostos um tanto espaçadamente, em linhas lonjitudinaes.

A superficie do corpo é coberta de salierıcias, analogas ás observadas na Vorticella monilata TATEM e dispostas em linhas lonjitudinaes. Essas saliencias só se encontram na parte media do corpo, faltando tanto na tromba como no prolongamento caudal. Năo foi possivel observar o macro e micro-nucleo.

Vacuolo contratil unico, situado na base do prolongamento caudal.
Dimensões: comprimento $140 \mu$, largura $20 \mu$.

Habitat: Encontrada em agua salôbra, nos arredores de Manguinhos. Muito raro.

Resumindo os resultados das pesquizas até agora efetuadas sobre a fauna de protozoarios do Brazil temos de acrecentar ás 251 especies assinaladas err nossa tese, 8 mencionadas em trabalho anterior, 23 na presente contribuição, sendo 22 dos arredores de Manguinhos e 1 de Angra dos Reis, 38 rejistadas por WAILES o que eleva a 320 o numero de protozoarios de vida livre até agora conhecidos no Brazil. 


\section{$-71-$ \\ Bibliografia.}

BLOCHMANN, F. 1895 Mikroskopische Tierwelt des Suesswassers. 1. Abt. Protozoa. (2. Afl.) Hamburg.

BUETSCHLI, O. 1887/9 Protozoa. In Bronn's Klassen u. Ordnungen des Tierreichs Bd. I. Lpz.

CLAPAREDE \& 1859/61 Etudes sur les infusoires et les rhizopodes. Genève.

\section{LACHMANN}

CUNHA, A. M.

CUNHA, A. M.

1915

CUNHA, A. M.

1915

DADAY, E.

1904

GOLDSCHMIDT, R. 1907

HAMMBURGER, C. \& BUDDENBROCK 1911

KLEBS, G. 1892

LAUTERBORN，R. 1908

LEMMERMANN, E. 1910

LEVANDER, R. M. 1894 MERMOD, G. 1914

PLAYFAIR, J. l. 1915 ROUX, J.
Tropidomonas rotans n. g. nl. sp. (Nota prévia). Brazil-Medico-Anno XXIX, No 15, p. 113.

Sobre duas novas especies de ciliados (Nota prévia). Brazil-Medico, Anno XXIX, No 17, p. 129.

Spirorhynchus verrucosus n. g. n. sp. (Nota prévia). Brazil-Medico, Anno XXIX, No 19, p. 145.

Suesswasser-Mikrofauna Paraguays. Protozoa.

Bibliotheca zoologica Heft 44 pp. 4-46, Taf. I. Stuttgart.

Lebensgeschichte der Mastigamoeben, Mastigella vitrea n. sp. und Mastigina setosa n. sp.

Arch. f. Protistenkunde, Supl. I. pp. 83-168, Taf. V-IX.

Nordische Ciliata mit Ausschluss der Tritinnoidea.

Nordisches Plankton hrsg. von Dr. Prof. K. BRANDT \& Prof. Dr. APSTEIN in Kiel. XIII.

Flagellaten-Studien.

Zeits. f. wiss. Zool. Bd. 55, pp. 265-351; 352-445. Taf. XIIIXVIII Lpz.

Protozoen-Studien v. Teil. Zur Kenntnis einiger Rhizopoden u. Infusorien aus dem Gebiete des Oberrheins. Zeits. f. wiss. Zoologie Bd. 90 pp. 645-669, Taf. 41-45 Lpz.

Algen I. (Schizophyten, Flagellaten u. Peridineen) Kryptogamenflora der Mark Brandenburg, Bd. III.

Beitrage zur Kenntnis einiger Ciliaten. Helsingfors.

Recherches sur la faune infusorienne des tourbières et des eaux voisines de Sainte Croix (Jura vaudois). Revue Suisse de Zoologie Vol. 22 u. 3. pp. 31-114. Pl. 2-3.

Freshwater Algae of the Lismore District. The Proc. of the Linn. Soc. of New South Wales. Vol. XL $\mathrm{n}$. 158. pp. 310-362 Pl. XL I-XLVl. Sydney.

Observations sur quelques infusoires ciliés des environs $d \epsilon$ Genève avec la description de nouvelles espèces Revue suisse de Zoologie Tome 6, pp. 557-635, pl. 13 \& 14 Geneve. 
rienne des eaux stagnantes des environs de Oene-

ROUX, J.

1901 ve, Genève.

SCHEWIAKOFF, W. 1896

Infusoria aspirotricha (Holotricha auctorum).

Mém. de l'Acad. des Sc. de S. Pétersbourg. Sér. VIII, T. IV No 1 p. 1-395 Taf. I-VIl. S. Pétersbourg.

SCHOUTEDEN, FR. 1906

Les Rhizopodes testacés d'eau douce d'après la monographie du Prof. A. AWERINZEW.

Annales de Biologie lacustre Tome I. pp. 327-382. Bruxelles.

SCHOUTEDEN, FR. 1906

Les infusoires aspirotriches d'eau douce.

Annales de Biologie lacustre Tome I. pp. 383-468.

STEIN, FR.

1859-83 Der Organismus der Infusionstiere. Lpz.

WAILES, G. H. 1913

Freshwater Rhizopoda from North and South America.

Journ. of the Linnean Soc. Zoology. Vol. XXXIl pp. 201 218 pl. 15. London. 



$$
-73
$$

\section{Esplicação das figuras.}

Todas as figuras foram desenhadas com camara clara á altura da mesa, sendo o comprimento do lubo do microscopio de $16 \mathrm{~cm}$, Ob. imersão homojenea 1/12, Oc. 2 de Zeiss.

Fig. 1. Tropidomonas rotans CUNHA 1915.

* 2. Metopus nasutus CUNHA 1915.

- 3. Metopus caudatus CUNHA 1915.

* 4. Spirorhynchus verrucosus CUNHA 1915. 